\title{
Chronic resveratrol administration improves diabetic cardiomyopathy in part by reducing oxidative stress
}

\author{
Majid Mohammadshahi ${ }^{1}$, Fatemeh Haidari ${ }^{2}$, Farhad Ghadiri Soufi ${ }^{3}$ \\ ${ }^{1}$ Hyperlipidemia Research Center, Ahvaz Jundishapur \\ University of Medical Sciences, Ahvaz, Iran \\ ${ }^{2}$ Nutrition and Metabolic Diseases Research Center, \\ Ahvaz Jundishapur University of Medical Sciences, Ahvaz, Iran \\ ${ }^{3}$ Molecular Medicine Research Center, \\ Hormozgan University of Medical Sciences, Bandar Abbas, Iran
}

\begin{abstract}
Background: Based on the key role of hyperglycemia-mediated oxidative stress in the pathogenesis of diabetic cardiomyopathy, increasing antioxidant defense would represent a novel therapeutic approach for management of diabetic cardiomyopathy. This study was designed to seek the effectiveness of chronic treatment with resveratrol, a potent natural antioxidant, on streptozotocin-nicotinamide experimental model of type 2 diabetic hearts.

Methods: Male rats randomized into four groups $(n=12)$ : control, diabetic, control + resveratrol, and diabetic + resveratrol.

Results: Four-month oral resveratrol administration to diabetic rats $(5 \mathrm{mg} / \mathrm{kg} /$ day) alleviated the reduction of cardiac antioxidant enzymes activities $(3.88 \pm 0.48 \mathrm{vs} .1 .49 \pm 0.43 \mathrm{U}, p<0.05$ for superoxide dismutase, and $2.72 \pm 0.26 \mathrm{vs} .1 .18 \pm 0.19 \mathrm{nmol} / \mathrm{min} / \mathrm{mL}, p<0.05$ for catalase) and the enhancement of cardiac oxidative markers (5.01 $\pm 0.37 \mathrm{vs} .7 .23 \pm 0.51 \mathrm{ng}$, $p<0.05$ for 8-isoprostane, $6.03 \pm 0.87$ vs. $8.49 \pm 0.52 \mu \mathrm{mol}, p<0.05$ for nitrite/nitrate, and $0.44 \pm 0.03$ vs. $0.59 \pm 0.04, p<0.05$ for oxidized/reduced glutathione ratio), nuclear factor kappa $B$ activity $(0.37 \pm 0.09$ vs. $0.60 \pm 0.11, p<0.05)$ and apoptosis rate $(0.98 \pm 0.28 v$ s. $1.63 \pm 0.16, p<0.05)$. Moreover, it improved left ventricular developed pressure $(72.46 \pm 8.16$ vs. $52.01 \pm 11.32 \mathrm{~mm} \mathrm{Hg}, p<0.05)$ and coronary flow $(14.08 \pm 1.09$ vs. $11.75 \pm 1.43 \mathrm{~mL} /$ Imin $\times g, p<0.05$ ).
\end{abstract}

Conclusions: These beneficial cardioprotective observations suggest that treatment with resveratrol can potentially delay or attenuate the progression of diabetes-related cardiac complications. (Cardiol J 2014; 21, 1: 39-46)

Key words: type 2 diabetes, antioxidant, hyperglycemia, redox status, cardiomyopathy, resveratrol

Address for correspondence: Dr Farhad Ghadiri Soufi (Assistant Professor of Physiology; PhD), Molecular Medicine Research Center, Hormozgan University of Medical Sciences, Jomhouri Boulevard, Bandar Abbas, Iran, tel/fax: +98 761333 7192, e-mail: Dr.F.G.Soufi@gmail.com 


\section{Introduction}

Diabetic cardiomyopathy has been defined as diabetes-mediated ventricular dysfunction independent of any vascular disease and hypertension, manifesting initially by diastolic dysfunction, later by systolic dysfunction, and finally by heart failure [1]. Diabetic cardiomyopathy is a common problem in diabetic population as it is detectable even in approximately $60 \%$ of well-controlled type 2 diabetic patients [1]. While several mechanisms have been discussed for onset and development of diabetic cardiomyopathy, it is believed that oxidative stress resulting from diabetes-mediated hyperglycemia, lipotoxicity and mitochondrial uncoupling play central roles in the pathogenesis of diabetic cardiomyopathy [2-4]. Oxidative stress, an imbalance between oxygen/nitrogen free radicals production and antioxidant defense, cause oxidation or glycation of lipids, proteins and DNA, and results in extracellular matrix change, small vessel disease, autonomic neuropathy and defects in cellular $\mathrm{Ca}^{2+}$ transport and contractile proteins. These complications, in turn, can result in cardiac hypertrophy, stiffness, fibrosis and apoptosis [1-3].

During recent years many investigations have been directed toward increasing antioxidant defense as a novel therapeutic approach against diabetes-related cardiovascular complications [1,2]. It has been widely accepted that resveratrol, a natural polyphenolic phytoalexin found in grapes, berries and peanuts, not only exerts potent antioxidant, anti-inflammatory, anti-apoptotic and some other cardioprotective effects in diabetic subjects, but also ameliorates diabetes-mediated hyperglycemia through insulin-dependent and insulin-independent pathways [5].

While there are sufficient evidences to support the protective role of resveratrol in different forms of heart diseases, there are few publications on the effects of resveratrol on diabetic cardiomyopathy and none of them have explored its long-term effects. For instance, it has been shown that resveratrol given at 7 and 15 days increased antioxidant proteins and reduced infarct size and apoptosis rate in diabetic rat heart [6, 7]. Another study has proven that treatment with resveratrol $(2.5 \mathrm{mg} / \mathrm{kg} /$ day orally for 2 weeks $)$ induced Glut-4 translocation and glucose uptake in diabetic rat myocardium [8]. Apart from that, Zhang et al. [9] have shown that treatment with resveratrol (20 mg/kg/day orally for 4 weeks) improves left ventricular (LV) diastolic relaxation by inhibiting oxidative/nitrosative stress. A very recent study has shown that 8 weeks treatment with resveratrol $(2.5 \mathrm{mg} / \mathrm{kg} /$ day; IP) improves diabetic heart fun- ction through reducing ventricular inflammation and remodeling [10]. In another recent study it has been shown that resveratrol given at $2.5 \mathrm{mg} /$ $/ \mathrm{kg} /$ day orally for 4 weeks prevented from cardiac and vascular dysfunction in diabetic rats through its effect on redox balance [5].

Most of researches on antidiabetic effects of resveratrol have been performed on animals, cell lines and tissue extracts and because of few performed clinical trials, scientists are not yet certain about sufficient efficacy of resveratrol in humans. On the other hand, since duration of diabetes is crucial for manifestation of some structural and functional complications of diabetic cardiomyopathy [1], the translation from animal studies to clinical trials needs further studies to clarify the particular effects of resveratrol.

Currently, resveratrol has become available in pill form as a dietary supplement and based on previous studies, it seems that prescription of resveratrol is useful, safe and well tolerated. Given the key role of oxidative stress in the pathogenesis of diabetic cardiomyopathy, we conducted the present study to evaluate the antioxidant effect of chronic resveratrol ingestion on oxidative stress, apoptosis rate and function of the heart in the experimental model of type 2 diabetic rats.

\section{Methods}

\section{Experimental design}

Male Sprague-Dawley rats (3 months old with 300-330 g of body weight; Razi Institute, Tehran, Iran) were housed in standard cages (3 rats in each), at room temperature $\left(22-25^{\circ} \mathrm{C}\right)$ with $12: 12$-h light/dark cycles and free access to food and water. Rats were randomly divided into four groups (12 in each): normal control (NC), diabetic control (DC), normal control treated with resveratrol (NTR), and diabetic treated with resveratrol (DTR). This study was designed in accordance with US National Institutes of Health (NIH publication, No. 86-23, revised 1996) and ARRIVE guidelines for the care and use of animals approved by the Ethics Committee for the Use of Animals in Research at Ahvaz Jundishapur University of Medical Sciences [11]. Type 2 diabetes was induced by injection of streptozotocin $(50 \mathrm{mg} /$ $/ \mathrm{kg}$; IP) dissolved in $0.1 \mathrm{M}$ of citrate buffer (pH 4.5), 15 min after the prescription of nicotinamide $(110 \mathrm{mg} / \mathrm{kg}$; IP) in $12 \mathrm{~h}$ fasted rats [12, 13]. Citrate buffer was injected alone in control rats. After $48 \mathrm{~h}$ blood glucose levels were measured using glucometer (Arkray, Kyoto, Japan) and the rats with blood glucose levels higher than $250 \mathrm{mg} / \mathrm{dL}$ were included to the protocol as diabetic rats [12]. All manipulations were held in the morning. 
Resveratrol treatment (Cayman chem., Ann Arbor, MI, USA) was carried out orally in aqueous solution for 4 months [12]. The dosage $(5 \mathrm{mg} / \mathrm{kg})$ was regulated every week. An oral glucose tolerance test (OGTT) was performed 1 day before killing the rats. OGTT first sample was taken from overnight fasted rats. Other blood samples were taken at 30,60,90, and 120 min intervals after intake of glucose solution at a dosage of $2 \mathrm{~g} / \mathrm{kg}$ of body weight. At the end of experimental period, fasted rats were anesthetized with pentobarbital sodium (35 mg/kg IP) and blood samples ( $3 \mathrm{~mL}$ per rat) were collected from retro-orbital sinus for determination of plasma insulin. After cervical decapitation, the hearts and pancreases were quickly removed. The hearts of 6 rats were used for evaluating functional parameters by Langendorff apparatus and the hearts of the other 6 rats were considered for determination of redox status, nuclear factor kappa B (NF- $\kappa \mathrm{B})$ activity and apoptosis rate. The pancreases were homogenized for determination of pancreatic insulin content.

\section{Heart preparation}

After anesthetization, the hearts were excised, placed in ice-cold saline, and rapidly hung by the aorta on the cannula of Langendorff apparatus, as we previously described [14]. A pressure-transducing catheter was placed through the cannula and aortic valve into the chamber of the $\mathrm{LV}$, and pressure was acquired with a computer connected to the transducer (Power-Lab, AD Instruments, Australia). After 5 min stabilization period, end diastolic and developed pressures were measured, and coronary flow rate was obtained by collecting the coronary effluent for $1 \mathrm{~min}$.

\section{Quantification of NF- $\kappa$ B activity}

Nuclear factor kappa B activity was determined using NF- $\kappa$ B p65 transcription factor assay kit (Cayman chem.) according to the procedures provided by the manufacturer. Briefly, $2 \mu \mathrm{g}$ of cardiac nuclear extracts were incubated with an oligonucleotide containing the NF- $\kappa \mathrm{B}$ consensus site, and then were incubated with monoclonal and secondary antibodies directed against the NF- $\kappa \mathrm{B}$ p65 subunit. Reaction was quantified at $450 \mathrm{~nm}$.

\section{Quantification of apoptosis}

Cell death detection ELISA kit (1544675, Roche, Germany) was used to detect the cytosolic histone-associated DNA fragmentation, based on the manufacturer's instructions. Cardiac cytoplasmic extracts $(25 \mu \mathrm{L})$ were used as an antigen source in a sandwich ELISA. The data were reported as an apoptotic index $\left(\mathrm{OD}_{405} / \mathrm{mg}\right.$ protein $)$ to indicate the apoptosis rate.

\section{Estimation of redox status in cardiac samples}

Superoxide dismutase (SOD) and catalase activities, oxidized glutathione/reduced glutathione (GSSG/GSH) ratio and the levels of nitrite/nitrate and 8-isoprostane were estimated in cardiac cytoplasmic extracts using colorimetric assay kits (Cayman chem.) according to the procedures provided by the manufacturer and were calculated from plotted standard curves at $450 \mathrm{~nm}$.

\section{Insulin measurements}

The plasma insulin concentration and the pancreatic insulin content were determined with rat ELISA kit (Cayman chem. 589501) according to the procedures provided by the manufacturer and were calculated from standard curves at $450 \mathrm{~nm}$.

\section{Data analysis}

Data were expressed as the mean $\pm \mathrm{SE}$ and were analyzed by repeated measure ANOVA for OGTT and one-way ANOVA for other parameters, using SPSS 21 software. When a significant p-value was obtained, the Tukey post-hoc test was employed to determine the differences between groups. A level of $\mathrm{p}<0.05$ was considered statistically significant.

\section{Results}

\section{Morphology and insulin level}

Table 1 shows that 4 months after diabetes induction, the diabetic rats in the DC and DTR groups show significant decline in the body weight compared to the NC group ( $\mathrm{p}<0.01$ for both); However, this weight loss was lower in the DTR group than the DC group ( $\mathrm{p}<0.05)$. We did not see a significant difference between the body weights of the NTR and NC groups. On the other hand, the heart weight increased significantly in the DC and DTR groups when compared with the NC group ( $\mathrm{p}<0.05$ for both), and 4 months' resveratrol administration could not exert significant effect on this variable.

Table 1 represents also that the fasted plasma insulin concentration in both diabetic groups was significantly lower than in the NC group $(\mathrm{p}<0.01$ for both) and resveratrol administration could not significantly change the insulin concentration in diabetic and non-diabetic rats. On the other hand, 
Table 1. Effect of chronic resveratrol administration on the body weight, heart weight and fasted insulin levels in the blood and pancreas of experimental groups.

\begin{tabular}{lcccc}
\hline & $\begin{array}{c}\text { Body weight } \\
{[\mathrm{g}]}\end{array}$ & $\begin{array}{c}\text { Heart weight } \\
{[\mathrm{g}]}\end{array}$ & $\begin{array}{c}\text { Plasma insulin } \\
{[\mathrm{pg} / \mathrm{mL} \text { ] }}\end{array}$ & $\begin{array}{c}\text { Pancreatic insulin } \\
{[\mathrm{ng} / \mathrm{mg} \text { protein] }}\end{array}$ \\
\hline NC & $493.46 \pm 25.16$ & $0.92 \pm 0.06$ & $52.81 \pm 7.95$ & $3.07 \pm 0.21$ \\
DC & $241.28 \pm 16.91^{*}$ & $1.42 \pm 0.08^{* *}$ & $15.42 \pm 3.86^{*}$ & $1.12 \pm 0.09^{*}$ \\
NTR & $481.71 \pm 28.18$ & $1.02 \pm 0.05$ & $48.61 \pm 9.37$ & $3.72 \pm 0.37$ \\
DTR & $301.14 \pm 17.54^{* \#}$ & $1.33 \pm 0.06^{* *}$ & $12.04 \pm 5.29^{*}$ & $2.68 \pm 0.26^{* \#}$ \\
\hline
\end{tabular}

The values represent the mean \pm SE of 12 animals per group; ${ }^{*} \mathrm{p}<0.01$ and ${ }^{*}{ }^{*} \mathrm{p}<0.05$ vs. normal control group (NC), respectively; $\# p<0.05$ vs. diabetic control group (DC); NTR - normal rats treated with resveratrol; DTR — diabetic rats treated with resveratrol

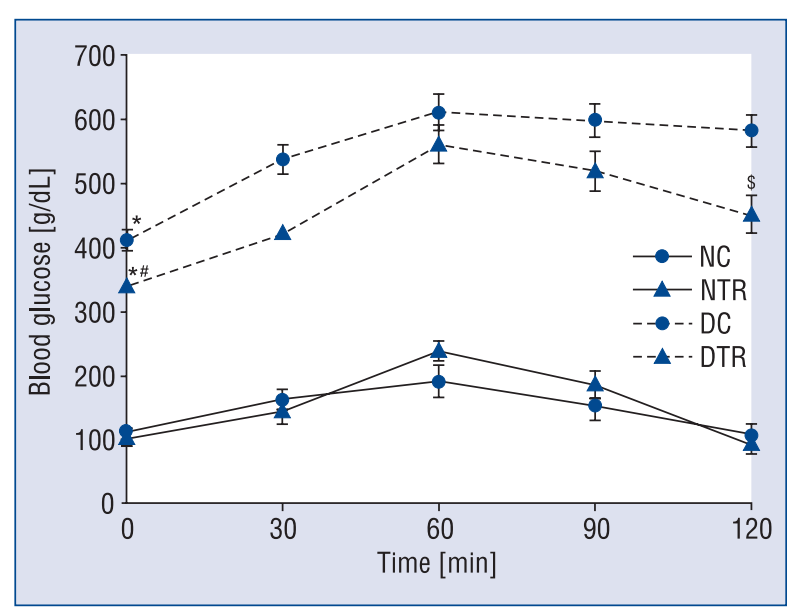

Figure 1. The results of oral glucose tolerance test at the end of protocol period ( $16^{\text {th }}$ week). The values represent the mean \pm SE of 12 animals per group; ${ }^{*} p<0.01$ vs. normal control group (NC); ${ }^{*} p<0.05$ vs. diabetic controls (DC); ${ }^{\$} \mathrm{p}<0.05$ vs. 60 min values; NTR - normal rats treated with resveratrol; DTR - diabetic rats treated with resveratrol.

while the pancreatic insulin content in the DC and DTR groups was lower than in the NC group ( $\mathrm{p}<0.01$ for both), four months treatment with resveratrol saved the pancreatic insulin content in the DTR group when compared with the DC group $(\mathrm{p}<0.05)$ (Table 1). No significant difference was observed between the pancreatic insulin content of the NC and NTR groups.

\section{OGTT}

Figure 1 depicts the results of OGTT, performed at the end of the $16^{\text {th }}$ week of the protocol. Although a significant hyperglycemia was observed at the baseline time (time 0 ) in both diabetic groups ( $p<0.01$ for both DC and DTR groups), the blood glucose concentration was lower in the DTR group than in the DC group $(\mathrm{p}<0.05)$. While the blood glucose concentration in all groups increased to a maximum value at $60 \mathrm{~min}$ after glucose intake and decreased at $120 \mathrm{~min}$, it did not revert to the baseline level in the DC and DTR groups. However, the blood glucose concentration in the DTR group at minute 120 was significantly lower than minute 60 values $(\mathrm{p}<0.05)$. This indicates that the rate of peripheral glucose uptake was higher in the DTR group than in the DC group. We did not see a significant change in the blood glucose concentration between the NTR and NC groups.

\section{Left ventricular pressures and coronary flow}

Figure 2 depicts that in comparison to the NC group, LV diastolic pressure was higher in both diabetic groups ( $p<0.01$ for both) and resveratrol administration could not alleviate the elevated level in diabetic rats. Although LV developed pressure and coronary flow were lower in both diabetic groups than in the NC group ( $p<0.01$ for the DC and $\mathrm{p}<0.05$ for the DTR group), 4 months' resveratrol administration markedly attenuated these abnormalities in the DTR group as compared with the DC group ( $\mathrm{p}<0.05$ for both comparisons). No significant differences were observed in the measured cardiac performance indices between the NTR and NC groups.

\section{Redox status, NF- $\kappa$ B activity and apoptosis rate}

Figure 3 represents the effect of chronic resveratrol administration on the cardiac oxidative markers (8-isoprostane, nitrite/nitrate and GSSG/ /GSH), antioxidant enzyme activities (SOD and catalase), NF- $\kappa$ B activity and apoptosis rate. In comparison to the normal control rats, treatment with resveratrol enhanced SOD activity and reduced GSSG/GSH ratio in the heart of NTR group rats ( $p<0.05$ for both comparisons), while it has no significant effect on the other parameters. We observed reduced antioxidant enzymes activities 


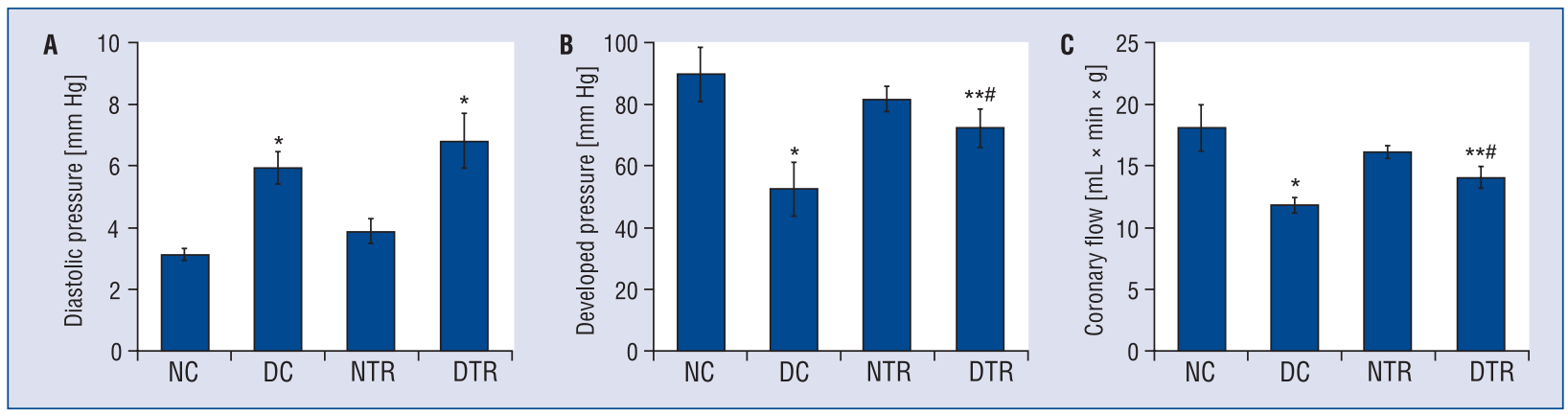

Figure 2. Effect of long-term treatment with resveratrol on the cardiac left ventricular diastolic pressure (A), left ventricular developed $(\mathbf{B})$ and coronary flow $(\mathbf{C})$. The values represent the mean \pm SE of 6 animals per group; ${ }^{*} p<0.01$; ${ }^{* *} p<0.05$ vs. normal control group (NC); \#p < 0.05 vs. diabetic control group (DC); NTR — normal rats treated with resveratrol, DTR — diabetic rats treated with resveratrol.

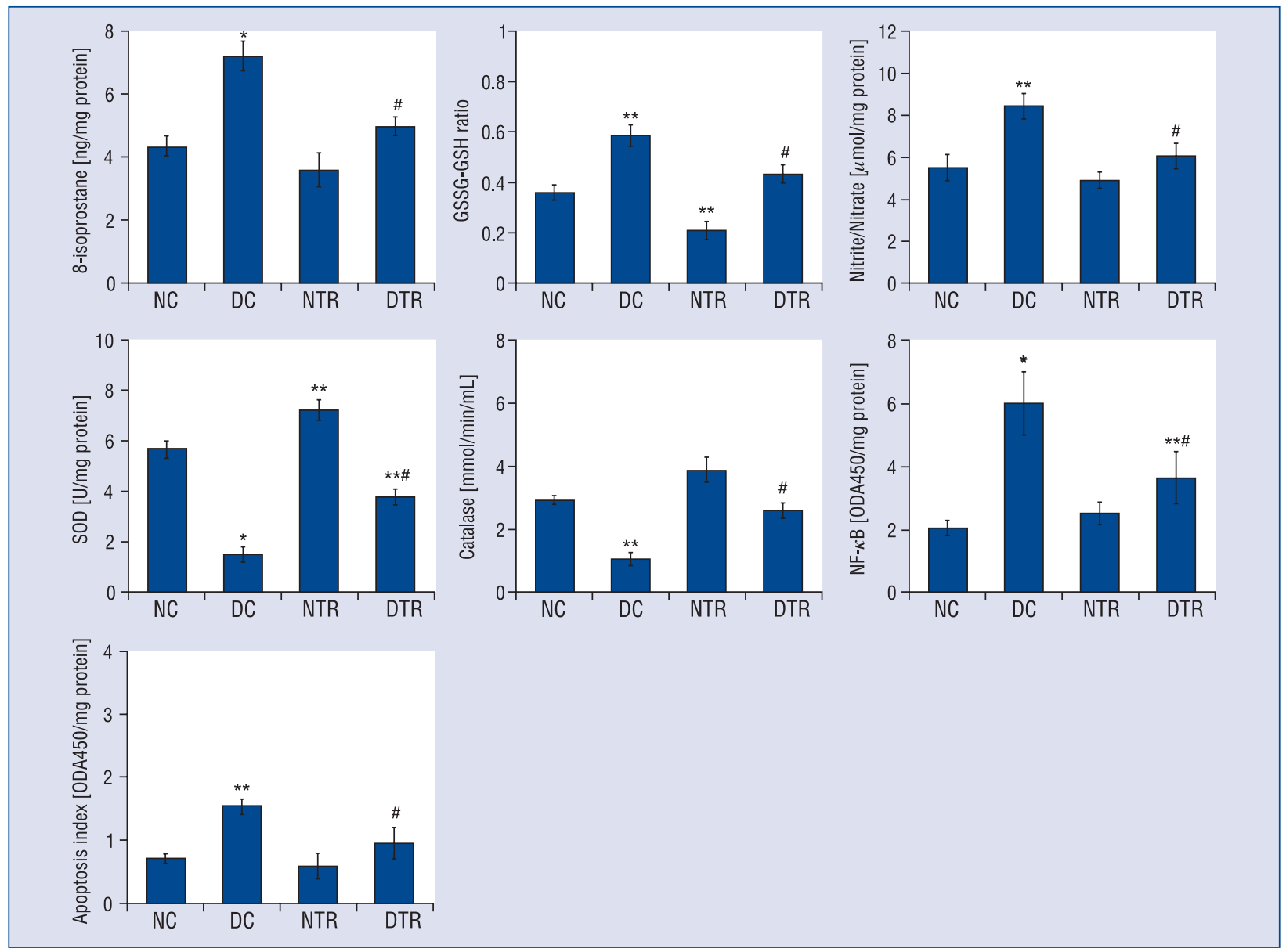

Figure 3. Effect of chronic resveratrol administration on the cardiac oxidative markers, apoptosis rate and the activity of antioxidant enzymes and NF- $\kappa$ B. The values represent the mean \pm SE of 6 animals per group; ${ }^{*} p<0.01$; ${ }^{* *} \mathrm{p}<0.05$ vs. normal control group (NC); \#p $<0.05$ vs. diabetic control group (DC); NTR — normal rats treated with resveratrol; DTR — diabetic rats treated with resveratrol; SOD - superoxide dismutase; GSSG/GSH — oxidized to reduced glutathione ratio.

( $p<0.01$ for SOD and $\mathrm{p}<0.05$ for catalase) with concomitant enhancement in nitrite/nitrate and 8 -isoprostane levels, GSSG/GSH ratio, NF- $\kappa \mathrm{B}$ activity and apoptosis rate in the heart of the DC group ( $\mathrm{p}<0.01$ for 8 -isoprostane and NF- $\kappa \mathrm{B}$ and $\mathrm{p}<0.05$ for the other parameters). All of these changes were significantly attenuated by chronic resveratrol administration (Fig. 3). 


\section{Discussion}

\section{Blood glucose and body weight}

Anti-hyperglycemic effect of resveratrol has been documented by several studies [5, 12, 13]. It is believed that resveratrol ameliorates diabetes-mediated hyperglycemia through insulin-dependent and insulin-independent pathways [5]. Szkudelski and Szkudelska [15] have reported that resveratrol preserves the pancreatic beta cells against chronic overstimulation leading to insulin secretion ability, at least for longer time. On the other hand, it has been shown that resveratrol increase the translocation of Glut- 4 and glucose uptake in diabetic peripheral tissues through activation of some intracellular signaling components via an insulin-independent manner $[8,16]$. In the present study, although resveratrol could not increase the blood insulin concentration in diabetic rats, it prevented from pancreatic insulin depletion and enhanced the peripheral glucose utilization. Improvement in glucose metabolism in turn prevents from degradation of proteins and lipids from their reservoirs and attenuates the body weight loss during diabetes [11, 12, 17]. Improvement in insulin sensitivity after 4 -week resveratrol administration has been also recently reported in type 2 diabetic patients, as well as in adults with impaired glucose tolerance [18, 19]. Moreover, Bhatt et al. [20] have shown that 3 months' supplementation of resveratrol in type 2 diabetic patients improved glycemic control with no significant change in body weight. On the other hand, Poulsen et al. [21] have proved that 4 weeks' treatment with resveratrol had no effect on resting energy expenditure and endogenous glucose turnover.

\section{Diabetic heart and oxidative stress}

During euglycemic condition, moderate amounts of reactive oxygen species (ROS) including superoxide $\left(\mathrm{O}_{2}{ }^{-}\right)$, hydrogen peroxide $\left(\mathrm{H}_{2} \mathrm{O}_{2}\right)$, and hydroxyl radical $\left(\mathrm{OH}^{*}\right)$ are produced in the cells and contribute to some physiological process. Dismutation of $\mathrm{O}_{2}{ }^{--}$to $\mathrm{H}_{2} \mathrm{O}_{2}$ by SOD, is the first step in ROS detoxification. Then, $\mathrm{H}_{2} \mathrm{O}_{2}$ is metabolized into water by the activities of catalase and glutathione peroxidase. Moreover, GSH, a co-substrate for glutathione peroxidase activity, is another major intra- and extracellular antioxidant molecule and acts as a direct free radical scavenger by conversion to GSSG [13]. GSSG to GSH ratio is a good marker for estimation of redox status [12,22].

Chronic hyperglycemia results in oxidative stress through direct generation of ROS and re- duction in the ability of the endogenous antioxidants to neutralize ROS, and/or by altering the redox balance [23]. This situation is traceable by measurements of lipid peroxidation, protein oxidation, reduction of antioxidant enzymes activities and cell death [12]. Also, nitric oxide (NO') can react with $\mathrm{O}_{2}{ }^{--}$to form a potent oxidizing agent, peroxynitrite $\left(\mathrm{ONOO}^{-}\right)$, and account for more cellular damage $[12,23]$. Due to very low concentration and short half-life of $\mathrm{NO}^{\circ}$, its stable metabolites such as nitrite $\left(\mathrm{NO}_{2}{ }^{-}\right)$and nitrate $\left(\mathrm{NO}_{3}{ }^{-}\right)$are determined as suitable indices for estimation of $\mathrm{NO}^{*}$ [24]. On the other hand, many of hyperglycemia-induced pathways converge to activate NF- $\kappa \mathrm{B}$, a proinflammatory master switch, which, in turn, contributes to increase of proinflammatory cytokine gene expressions, overproduction of $\mathrm{NO}^{\circ}$, oxidative stress worsening and apoptosis propelling in the heart [25].

In this context, our data are in agreement with the previous studies, in which our diabetic control rats experienced chronic hyperglycemia with reduction in SOD and catalase activity with concomitant enhancements in the cardiac oxidative stress markers (including 8-isoprostane, nitrite/ nitrate and GSSG /GSH ratio), NF- $\kappa$ B activity and apoptosis rate.

\section{Resveratrol and cardioprotection}

Antioxidant and cardioprotective properties of resveratrol have been documented by a variety of animal studies and human clinical trials [2, 5, $26,27]$. In this context, it has been reported that resveratrol administration in patients with stable coronary artery disease $(8-10 \mathrm{mg} / \mathrm{kg}$ for 3 and 12 months), improved LV diastolic pressure and circulatory proinflammatory markers respectively [26, 27]. Also, Militaru et al. [28] have shown that 60 days' treatment with resveratrol decreased inflammatory markers in patients with stable angina pectoris.

The beneficial effects of resveratrol with different dosage $(2.5-20 \mathrm{mg} / \mathrm{kg})$ in diabetic hearts have been previously reported by several animal studies and none of them have explored the effect of resveratrol for more than 2 months $[5,7,9]$. Thirunavukkarasu et al. [7] have shown that 15 days' treatment with resveratrol $(2.5 \mathrm{mg} / \mathrm{kg} /$ day $)$ reduced cardiac apoptosis rate and improved LV developed pressure and coronary flow in diabetic rats through enhancement in $\mathrm{NO}^{\circ}$ production and SOD activity. In addition, Zhang et al. [9] have demonstrated that resveratrol administration for 4 weeks $(20 \mathrm{mg} / \mathrm{kg}$ ) /day) improved LV diastolic relaxation by stabili- 
zation of redox status and inhibiting the activity of NF- $\kappa$ B. In a recent study, Turan et al. [5] have shown that resveratrol given at $2.5 \mathrm{mg} / \mathrm{kg} /$ day orally for 4 weeks improved coronary perfusion pressure and heart rate in diabetic rats through reducing soluble sulfhydryl, nitrite and thioredoxin reductase levels and improvement in redox status. To the best of our knowledge, there is no published paper on the role of resveratrol in patients with diabetic cardiomyopathy.

The antioxidant property of resveratrol has been suggested to be performed directly by its free radical quenching action and by modulating the activation of NF- $\kappa \mathrm{B}$, proinflammatory mediators and antioxidant enzymes at transcriptional or post-transcriptional levels, or indirectly by reducing hyperglycemia [9, 16, 29-31].

In this context, our data are in accordance with the results from literature obtained from short-term resveratrol administration in diabetic hearts in which 4-month oral resveratrol intake ( $5 \mathrm{mg} / \mathrm{kg} /$ day; orally) increased SOD and catalase activity, reduced oxidative stress markers (8-isoprostane, nitrite/nitrate and GSSG/GSH ratio), NF- $\kappa$ B activity and apoptosis rates in diabetic rats. Moreover, we showed that resveratrol improved LV developed pressure and coronary flow, but it could not affect the LV diastolic pressure significantly. While abnormal diastolic function is recognized as the earliest manifestation of diabetic cardiomyopathy, it is believed that the appearance of systolic dysfunction depends on duration of diabetes [1]. Hence, it is expectable that because of the long-term duration of uncontrolled diabetes in the present study, the diastolic dysfunction might be irreversible by resveratrol treatment.

\section{Conclusions}

In conclusion, our results depict that chronic resveratrol administration has an anti-hyperglycemic effect in diabetic rats. Moreover, it alleviates the impairment of cardiac performance in part by reducing oxidative stress and apoptosis rate. While there is sufficient evidence from intact organ and animal studies to support the cardioprotective role of resveratrol in different forms of heart diseases, there are very few papers published on the effects of resveratrol on human heart and none of them have investigated its role on diabetic cardiomyopathy. Our observations may suggest that therapies during early stages of diabetes with antioxidant and anti-hyperglycemic agents such as resveratrol can potentially delay or attenuate the progression of diabetic cardiomyopathy but further human clinical trials are needed to confirm this hypothesis.

\section{Acknowledgements}

The grant of this study was supported by Nutrition \& Metabolic Diseases Research Center, Ahvaz Jundishapur University of Medical Sciences, Ahvaz, Iran (Grant No: NRC-9007).

Conflict of interest: none declared

\section{References}

1. Falcão-Pires I, Leite-Moreira AF. Diabetic cardiomyopathy: Understanding the molecular and cellular basis to progress in diagnosis and treatment. Heart Fail Rev, 2012; 17: 325-344.

2. Xu YJ, Tappia PS, Neki NS, Dhalla NS. Prevention of diabetes-induced cardiovascular complications upon treatment with antioxidants. Heart Fail Rev, 2014; 19: 113-121.

3. Khullar M, Al-Shudiefat AA, Ludke A, Binepal G, Singal PK. Oxidative stress: A key contributor to diabetic cardiomyopathy. Can J Physiol Pharmacol, 2010; 88: 233-240.

4. Csiszar A. Anti-inflammatory effects of resveratrol: Possible role in prevention of age-related cardiovascular disease. Ann N Y Acad Sci, 2011; 1215: 117-122.

5. Turan B, Tuncay E, Vassort G. Resveratrol and diabetic cardiac function: Focus on recent in vitro and in vivo studies. J Bioenerg Biomembr, 2012; 44: 281-296.

6. Dekkers DH, Bezstarosti K, Gurusamy N et al. Identification by a differential proteomic approach of the induced stress and redox proteins by resveratrol in the normal and diabetic rat heart. J Cell Mol Med, 2008; 12: 1677-1689.

7. Thirunavukkarasu M, Penumathsa SV, Koneru S et al. Resveratrol alleviates cardiac dysfunction in streptozotocin-induced diabetes: Role of nitric oxide, thioredoxin, and heme oxygenase. Free Radic Biol Med, 2007; 43: 720-229.

8. Penumathsa SV, Thirunavukkarasu M, Zhan L et al. Resveratrol enhances GLUT-4 translocation to the caveolar lipid raft fractions through AMPK/Akt/eNOS signalling pathway in diabetic myocardium. J Cell Mol Med, 2008; 12: 2350-2361.

9. Zhang H, Morgan B, Potter BJ et al. Resveratrol improves left ventricular diastolic relaxation in type 2 diabetes by inhibiting oxidative/nitrative stress: in vivo demonstration with magnetic resonance imaging. Am J Physiol Heart Circ Physiol, 2010; 299: H985-H994.

10. Delucchi F, Berni R, Frati C et al. Resveratrol treatment reduces cardiac progenitor cell dysfunction and prevents morpho-functional ventricular remodeling in type-1 diabetic rats. PLoS One, 2012; 7: e39836.

11. Kilkenny C, Browne WJ, Cuthill IC, Emerson M, Altman DG. Improving bioscience research reporting: the ARRIVE guidelines for reporting animal research. PLoS Biol, 2010; 8: e1000412.

12. Soufi FG, Vardyani M, Sheervalilou R, Mohammadi M, Somi MH. Long-term treatment with resveratrol attenuates oxidative stress pro-inflammatory mediators and apoptosis in streptozotocin-nicotinamide-induced diabetic rats. Gen Physiol Biophys, 2012; 31 : 431-438.

13. Palsamy P, Subramanian S. Resveratrol, a natural phytoalexin, normalizes hyperglycemia in streptozotocin-nicotinamide induced experimental diabetic rats. Biomed Pharmacother, 2008; 62: 598-605.

14. Soufi FG, Saber MM, Ghiassie R, Alipour M. Role of 12-week resistance training in preserving the heart against ischemia-reperfusion-induced injury. Cardiol J, 2011; 18: 140-145. 
15. Szkudelska K, Szkudelski T. Resveratrol, obesity and diabetes. Euro J Pharmachol, 2010; 635: 1-8.

16. Lekli I, Szabo G, Juhasz B et al. Protective mechanisms of resveratrol against ischemia-reperfusion-induced damage in hearts obtained from Zucker obese rats: the role of GLUT- 4 and endothelin. Am J Physiol Heart Circ Physiol, 2008; 294: H859-H866.

17. Roghani M, Baluchnejadmojarad T. Mechanisms underlying vascular effect of chronic resveratrol in streptozotocin-diabetic rats. Phytother Res, 2010; 24: S148-S154.

18. Crandall JP, Oram V, Trandafirescu G et al. Pilot study of resveratrol in older adults with impaired glucose tolerance. J Gerontol A Biol Sci Med Sci, 2012; 67: 1307-1312.

19. Brasnyó P, Molnár GA, Mohás $M$ et al. Resveratrol improves insulin sensitivity, reduces oxidative stress and activates the Akt pathway in type 2 diabetic patients. Br J Nutr, 2011; 106: 383-389.

20. Bhatt JK, Thomas S, Nanjan MJ. Resveratrol supplementation improves glycemic control in type 2 diabetes mellitus. Nutr Res, 2012; 32: 537-541.

21. Poulsen MM, Vestergaard PF, Clasen BF et al. High-dose resveratrol supplementation in obese men: An investigator-initiated, randomized, placebo-controlled clinical trial of substrate metabolism, insulin sensitivity, and body composition. Diabetes, 2012; 62: 1186-1195.

22. Palsamy P, Subramanian S. Ameliorative potential of resveratrol on proinflammatory cytokines, hyperglycemia mediated oxidative stress, and pancreatic $\beta$-cell dysfunction in streptozotocin-nicotinamide-induced diabetic rats. J Cell Physiol, 2010; 224: 423-432.

23. Rains JL, Jain SK. Oxidative stress, insulin signaling, and diabetes. Free Radic Biol Med, 2011; 50: 567-575.
24. Pitocco D, Zaccardi F, Di Stasio E et al. Oxidative stress, nitric oxide, and diabetes. Rev Diabet Stud, 2010; 7: 15-25.

25. Lorenzo O, Picatoste B, Ares-Carrasco S, Ramírez E, Egido J, Tuñón J. Potential role of nuclear factor $\kappa \mathrm{B}$ in diabetic cardiomyopathy. Mediators Inflamm, 2011; 2011: 652097.

26. Tomé-Carneiro J, Gonzálvez M, Larrosa M et al. One-year consumption of a grape nutraceutical containing resveratrol improves the inflammatory and fibrinolytic status of patients in primary prevention of cardiovascular disease. Am J Cardiol, 2012; 110: 356-363.

27. Magyar K, Halmosi R, Palfi A et al. Cardioprotection by resveratrol: A human clinical trial in patients with stable coronary artery disease. Clin Hemorheol Microcirc, 2012; 50: 179-187.

28. Militaru C, Donoiu I, Craciun A, Scorei ID, Bulearca AM, Scorei RI. Oral resveratrol and calcium fructoborate supplementation in subjects with stable angina pectoris: Effects on lipid profiles, inflammation markers, and quality of life. Nutrition, 2013; 29: 178-183.

29. Akar F, Pektas MB, Tufan C et al. Resveratrol shows vasoprotective effect reducing oxidative stress without affecting metabolic disturbances in insulin-dependent diabetes of rabbits. Cardiovasc Drugs Ther, 2011; 25: 119-131.

30. Yun JM, Chien A, Jialal I, Devaraj S. Resveratrol up-regulates SIRT1 and inhibits cellular oxidative stress in the diabetic milieu: mechanistic insights. J Nutr Biochem, 2012; 23: 699-705.

31. Lee JH, Song MY, Song EK et al. Overexpression of SIRT1 protects pancreatic $\beta$-cells against cytokine toxicity by suppressing the nuclear factor-kappa B signaling pathway. Diabetes, 2009; 58 : 344-351. 